\title{
Osteogenic induction of bone marrow mesenchymal cells on electrospun polycaprolactone/chitosan nanofibrous membrane
}

\author{
Ying $\mathrm{HE}^{1,2}$, Wei WANG ${ }^{3}$, Xuyan TANG ${ }^{1}$ and Xin $\mathrm{LIU}^{4}$ \\ ${ }^{1}$ Key Lab of Oral Diseases Research of Anhui Province, Stomatologic Hospital \& College, Anhui Medical University, Anhui 230032, China \\ ${ }^{2}$ Department of Stomatology, The Second People's Hospital of Wuhu, Anhui 241000, China \\ ${ }^{3}$ Surgical Department one, Wuhu Traditional Chinese, Anhui 241000, China \\ ${ }^{4}$ Shanghai Biomaterials Research \& Testing Center, Ninth People's Hospital, Shanghai Jiaotong University School of Medicine, Shanghai 200023, \\ China \\ Corresponding author, Xin LIU; E-mail: liuxin0556@163.com
}

\begin{abstract}
A novel chitosan/polycaprolactone (CS/PCL) nanofibrous membrane by electrospinning was developed for guided tissue regeneration (GTR) to improve mechanical properties and to promote osteogenic differentiation. Firstly, chitosan and PCL solutions of different weight ratios $(0 / 100,30 / 70,50 / 50)$ were mixed and then electrospun. Our data demonstrated that the CS/PCL (30/70) nanofibrous membrane promoted an increased rBMSCs proliferation when compared to the CS/PCL (50/50) membrane and pure PCL (0/100) membrane. The highest ALP activity and extracellular calcium deposit were observed on the CS/PCL (30/70) nanofibrous membrane, followed by the CS/PCL (50/50) and pure PCL nanofibrous membrane. Furthermore, the expression of osteocalcin (OCN) and Runx2 were also significantly higher on the CS/PCL (30/70, 50/50) nanofibrous membrane as compared to the pure PCL nanofibrous membrane. In conclusion, the electrospun CS/PCL nanofibrous membrane was found to be a biocompatible material that could stimulate osteogenic differentiation, suggesting that the novel CS/PCL membrane has an interesting potential as use for GTR.
\end{abstract}

Keywords: Electrospun, Chitosan, Polycaprolactone, Bone marrow mesenchymal stem cell, Osteogenic differentiation

\section{INTRODUCTION}

Periodontal disease is one of the two main human oral diseases, which has become the prime reason of adult tooth loss and if not effectively controlled, it may lead to other systemic diseases ${ }^{1)}$. Periodontal tissue regeneration (GTR) technology has been widely accepted as an effective way to solve this problem. Therefore, the synthetic GTR membrane has garnered increasing attention in recent years. This barrier membrane's main function is to impede extension growth of fibroblasts and rapid proliferation of epithelial cells, thus allowing for bone marrow mesenchymal cell growth and adhesion, ultimately promoting renovation of periodontium defects and subsequent extracellular matrix deposition and bone mineralization. The required characteristics of GTR barrier membrane include good biocompatibility, absorbability, biological activity and having no maintenance of cell toxicity. At present, some non-degradable polymer materials such as polytetrafluoroethylene (PTFE) have been safely used in the clinical setting as a barrier membrane. However, the PTFE membrane did not degrade in vivo and there was need for a second surgery, which increased surgical trauma ${ }^{2}$. Therefore, researchers started paying close attention to the study of biodegradable materials including synthetic polymer [polylactic acid, poly glycolic acid, poly(Dl-Lactide-Co-Glycolide)] or natural polymers (collagen, chitosan). The biodegradable membrane

Color figures can be viewed in the online issue, which is available at J-STAGE.

Received May 28, 2016: Accepted Oct 24, 2016

doi:10.4012/dmj.2016-203 JOI JST.JSTAGE/dmj/2016-203 could undergo degradation and absorption slowly after implantation in vivo and therefore not require a second surgery for removal, thus reducing any surgical trauma or risk of failure. However, there are still some drawbacks for these biodegradable membranes such as low mechanical strength, lack of osteogenic abilities or potential immunogenicity (e.g. Collagen $)^{3,4)}$.

Recent years, chitosan (CS) and poly(caprolactone) have attracted particular attention in the field of tissue engineering for use as potential materials. Chitosan, a fully or partially deacetylated chitosan, has been used in many biomedical applications, such as wound dressings, drug-delivery systems and as nerve regeneration agents $^{5}$. These uses were made possible due to its high biocompatibility, low toxicity, biodegradability, bone regeneration ${ }^{6)}$, antimicrobial properties and no immune reaction ${ }^{7,8}$. Despite chitosan having potentially global value, however, its features of high brittleness, poor mechanical property, plasticity and insolubility in common organic solvents have hindered its basic research and applications in the clinical setting. Polycaprolactone (PCL), a semi-crystalline biodegadable polyester, has received U.S Food and Drug Administration approval for several clinical applications in the human body ${ }^{9}$. Consequently, it was also widely used for tissue engineering due to high plasticity, good mechanical properties and nontoxicity ${ }^{10)}$. However, the shortcomings of PCL, such as its slow degradation, strong hydrophobicity and lack of bioactive functions have greatly restricted its employment in tissue engineering. Recently, to 
combine the high plasticity and strength of PCL and the excellent biological properties of CS, some researchers have attempted to blend the CS and PCL for wound healing, vascular graft, retinal or cartilage tissue regeneration and showed that the CS/PCL composition could support the proliferation of endothelial cells, promote wound healing and tissue regeneration ${ }^{11-15)}$. Particularly, some studies found that PCL/CS scaffolds containing $20 \%$ chitosan prepared by melt stretching and multilayer deposition (MSMD) technique had rather low efficacy for repairing bone defects ${ }^{13)}$, while other studies showed that scaffolds with a shish-kebab (SK) structure formed by poly(e-caprolactone) (PCL) nanofibers and chitosan-PCL (CS-PCL) copolymers had good potential for bone tissue engineering ${ }^{16)}$. However, up to date, few studies focused on the effects of CS/PCL blends on repairing periodontal defects, it is unclear whether CS/PCL nanofibrous membrane could promote periodontal tissue regeneration, and the related mechanism are still lacking.

In the present study, a novel CS/PCL nanofibrous membrane with different weight ratio $(0 / 100,30 / 70$, 50/50) combining advantages of good biocompatibility of CS and high mechanical property of PCL has been developed by electrospinning. Meanwhile, the surface topography was characterized. In addition to good biological compatibility and mechanical strength, favorable GTR materials should have the ability of improving the adhesion and osteogenic induction of bone marrow-derived mesenchymal stem cells (BMSCs). Herein, BMSCs were cultured on the new CS/PCL membrane and the adhesion, proliferation and differentiation of cells on the membranes were evaluated to provide a scientific basis for its clinical use in periodontal tissue regeneration.

\section{MATERIALS AND METHODS}

\section{Membrane fabrication and characterization}

Pure PCL membrane: PCL (Sigma, St. Louis, MO, USA) weighing $1.5 \mathrm{~g}, 5 \mathrm{~mL}$ acetone stirred for $3 \mathrm{~h}$ until a transparent sticky solution is obtained. The solution was placed in a $5 \mathrm{~mL}$ injector, we chose a $20 \mathrm{G}$ nozzle which was connected to the positive voltage, the receiver was connected with to the negative voltage. Spinning conditions were as follows: $10 \mathrm{kV}$ positive voltage, $2 \mathrm{kV}$ negative voltage, the distance between the nozzle and the receiver was $15 \mathrm{~cm}$ with $2 \mathrm{~mL} / \mathrm{h}$ solution flowing. Operating temperature was same as room temperature at $27^{\circ} \mathrm{C}$. CS/PCL membrane: PCL weighing $1 \mathrm{~g}, \mathrm{CS}$ (Sigma) was added after weighing proportionally. Five milliliter of HFIP solvent (Sigma) was added with stirring for $10 \mathrm{~h}$ until a transparent sticky solution was obtained. The solution was placed in the $5 \mathrm{~mL}$ injector, we chose the $20 \mathrm{G}$ nozzle which was connected to the positive voltage, and the receiver was connected to the negative voltage. The spinning conditions were as follows: $12 \mathrm{kV}$ positive voltage, $2 \mathrm{kV}$ negative voltage, the distance between the nozzle and the receiver was 15 $\mathrm{cm}$ with $2 \mathrm{~mL} / \mathrm{h}$ solution flowing. Operating temperature was $27^{\circ} \mathrm{C}$. Subsequently, the morphology of different electrospun nanofibrous membranes was analyzed with a scanning electron microscopy (SEM, JSM-6700F, JEOL, Tokyo, Japan).

\section{Primary culture and subculture of rBMSCs}

The experimental animals were SD rats aged between 4 to 6 weeks, weighing $50-70 \mathrm{~g}$ and both males and female rats were included in the study. They were provided by the animal experimental center of Anhui Medical University. Rat bone marrow-derived mesenchymal stem cells (rBMSCs) were acquired from the femora of 5 -week-old SD rats. The marrow of the femora was rushed out with $10 \%$ FBS medium and suspended in L-DMEM supplemented with 10\% fetal bovine serum (FBS, Hyclone, Logan, UT, USA), 100 $\mathrm{mg} / \mathrm{L}$ streptomycin (Hyclone) And $100 \mathrm{U} / \mathrm{mL}$ penicillin. The whole bone marrow cells adherent culture method was used at a constant temperature of $37^{\circ} \mathrm{C}$. Culture was undertaken in a $5 \% \mathrm{CO}_{2}$ saturated humidity incubator. Non-adherent cells were dislodged after 3 days. New medium was replaced every 2-3 days, while observing adherence and growth of rBMSCs under an inverted microscope. When approximately $80 \%$ confluence was achieved, rBMSCs were passaged and used in the following experiments from the third to fifth passages.

The different electrospun nanofibrous membranes were sterilized under an ultraviolet lamp for $30 \mathrm{~min}$ at room temperature and placed in 24-well culture plates. Fourth generation rBMSCs were attained and centrifugated before seeding into 24 -well culture plates with and without nano fibrous membranes. The nonadherent cells were discarded after 1 day and 10\% FBS medium was replaced every $2-3$ days before cells overgrew.

\section{Morphology of rBMSCs on electrospun nanofibrous membranes \\ Morphology of rBMSCs on electrospun nano fibrous membranes was observed by using SEM. After 4 days, the cell-cultured membranes were fixed in $4 \%$ glutaraldehyde for $2 \mathrm{~h}$ at $4^{\circ} \mathrm{C}$. Following rinsing three times with PBS solution, the samples were dehydrated through a series of graded ethanol $(40,50,60,70,80$, 90, 95 and 100\%) and then dried. Finally, they were observed with SEM (JSM-6700F, JEOL).}

\section{Cell viability assays}

The proliferation of rBMSCs on the membranes of different proportion was investigated by using 3-(4,5-dimethylthiazol-2-yl)-2,5-diphenyltetrazolium bromide (MTT) colorimetric assays. The cells were seeded at $4.2 \times 10^{4} \mathrm{cells} / \mathrm{cm}^{2}$ in 24 -well plates with and without membranes of different proportion. They were cultured up to 1 st, $3 \mathrm{rd}$, 5 th and 7 th day. Then, they were rinsed with PBS three times and incubated in MTS reagent for $4 \mathrm{~h}$ at the constant temperature of $37^{\circ} \mathrm{C}$. They were cultured in a $5 \% \mathrm{CO}_{2}$ saturated humidity incubator. After $4 \mathrm{~h}$, MTT was drained from every well and afterwards they were placed into Dimethyl sulfoxide 
(DMSO, Sigma). Ultimately, the incubated suspension was then placed into a 96 -well plate, and the absorbance was read at $490 \mathrm{~nm}$ using a microplate reader (ELX800; BioTeK, Winooski, VT, USA).

\section{DAPI fluorescence staining}

The cells were seeded at $4.2 \times 10^{4} \mathrm{cells} / \mathrm{cm}^{2}$ in 24 -well plates with membranes of different proportion. Firstly, they were rinsed with PBS one time. Then, they were fixed with $4 \%$ poly formaldehyde at room temperature for $20 \mathrm{~min}$. They were rinsed with PBS three times again. Secondly, dealing with $0.5 \%$ Triton-100 PBS standing at room temperature for $15 \mathrm{~min}$, they were rinsed with PBS three times again. Thirdly, adding $0.1 \mathrm{ug} / \mathrm{mL}$ diluent of DAPI to the holes with avoiding light $10 \mathrm{~min}$. In the end, they were rinsed with PBS three times.

\section{Alkaline phosphatase (ALP) activity assay}

ALP activity evaluation was performed using ALP Detection Kit (Jiancheng Technology, Nanjing, China). Firstly, rBMSCs were seeded in 24-well plates with and without nanofibrous membranes for 7 and 14 days respectively. Then, ALP activity was detected according to the manufacturer's instructions and the total protein content was examined using the BCA method.

\section{Alizarin Red S staining}

Alizarin Red $\mathrm{S}$ staining was used to observe extracellular matrix calcification. $\mathrm{rBMSCs}$ were seeded in 24-well plates with and without nano fibrous membranes and cultured for 21 days. Cells were fastened in $4 \%$ paraformaldehyde for $15 \mathrm{~min}$, and then stained in 1\% (w/v) Alizarin Red S (Shanghai Sai'ai Si Reagent Limited Company, Shanghai, China) (pH 4.14.5) for 20 min at room temperature.

\section{Western blot}

Total cellular proteins were extracted from the nanofibrous membranes with rBMSCs for 4 days. The specific operation is as follows: the samples were lysed in ice-cold lysis buffer [0.1\% sodium dodecyl sulfate (SDS), $50 \mathrm{~mm}$ Tris-HCl, 1\% NP-40, $150 \mathrm{~mm} \mathrm{NaCl}$ (Applygen, Beijing, China)] containing phosphatase inhibitor cocktail (Sigma) and 1 MM PMSF (phenylmethylsulphonyl fluoride) (Sigma) for $30 \mathrm{~min}$. Then, cell lysates (40-80 ug protein) were loaded onto SDS-polyacrylamide gels (8-12\% separation gels) and transferred onto nitrocellulose (NC) membranes (Amersham Biosciences, Piscataway, NJ, USA). The membrane was closed for $2 \mathrm{~h}$ with skim milk powder at room temperature. After which the membrane was incubated overnight at $4^{\circ} \mathrm{C}$ with anti-RUNX-2 (1:500, a mouse monoclonal antibody, Abcam, Cambridge, MA, USA), anti-OCN (1:10000, a mouse monoclonal antibody, Abcam) and $\beta$-actin (1:2000, a mouse monoclonal antibodies, Abcam). Then it was washed three times in Tris-buffered saline (TBS) containing $0.05 \%$ Tween-20 (TBST) before incubating the secondary antibodies at room temperature for $2 \mathrm{~h}$. Then, it was washed three times in TBS containing $0.05 \%$
Tween-20 (TBST) and one time in TBS without $0.05 \%$ Tween-20. Finally, the immuno-reactive bands were visualized with the ECL chemiluminescence reagent (Millipore, Billerica, MA, USA).

\section{Cell barrier function testing for nanofibrous membrane}

To test the cell barrier function of these nanofibrous membrane, we evaluated the effects of the novel membranes on cell migration using a fibroblast cell line (L929 cell) according to a previous published paper ${ }^{17}$. The cells were cultured in MEM containing 2 or $10 \%$ FBS in a double chamber dish divided by a porous membrane (Transwell cell culture inserts, Corning, NY, USA). The cells were inoculatedat a density of $2.5 \times 10^{5}$ cells/well in the upper chamber (containing $2 \% \mathrm{FBS}$ ) with three nanofibrous membranes placed at the bottom of the cell insert. Blank experiments were performed as controls without placing nanofibrous membranes at the bottom of the cell culture insert. Cell migration through the membrane after $24 \mathrm{~h}$, caused by the gradient of FBS concentration in the upper (2\%) and lower $(10 \%)$ chamber, was determined by measuring the OD values of viable cells in the lower chamber with the cell counting kit-8 (CCK-8) assay (Beyotime Institute of Biotechnology, Beijing, China).

\section{Statistical analysis}

In this study, all data presented in the experiment is represented as mean \pm standard deviation (SD). Every experiment was repeated at least three times. $p<0.05$ was taken as statistically significant and statistically significant differences among the various groups were measured using one-way ANOVA and $t$-test. All statistical analysis was carried out using SPSS 17.0 software.

\section{RESULTS}

\section{Membrane characterization}

Three kinds of membranes were visible as a white film-like substance. Figure 1 shows SEM micrographs of the membranes with different ratios of CS/PCL. It showed that there were even spinning and staggered arrangements inside the fiber membrane. There were pores of different sizes between the fibers, suggesting it can provide a larger surface area and space which is more conducive to cell adhesion and proliferation. Specifically, at a ratio of CS/PCL 0:100, the morphology and texture of the CS/PCL (0:100) membrane were uniform and the fibers were aligned neatly (Fig. 1A). When added into CS, as shown in Fig. 1B, nodules were formed between the fibers in the CS/PCL (50:50) membrane. As the proportion of PCL solution increased, the size of the nodules became smaller and fibers could become more homogeneous in the CS/PCL (30:70) membrane (Fig. 1C).

The culture and adhesion of rBMSCs on the membranes Figure 2 displays morphology of cultured rBMSCs in the three kinds of nanofibrous membranes. Cells were 

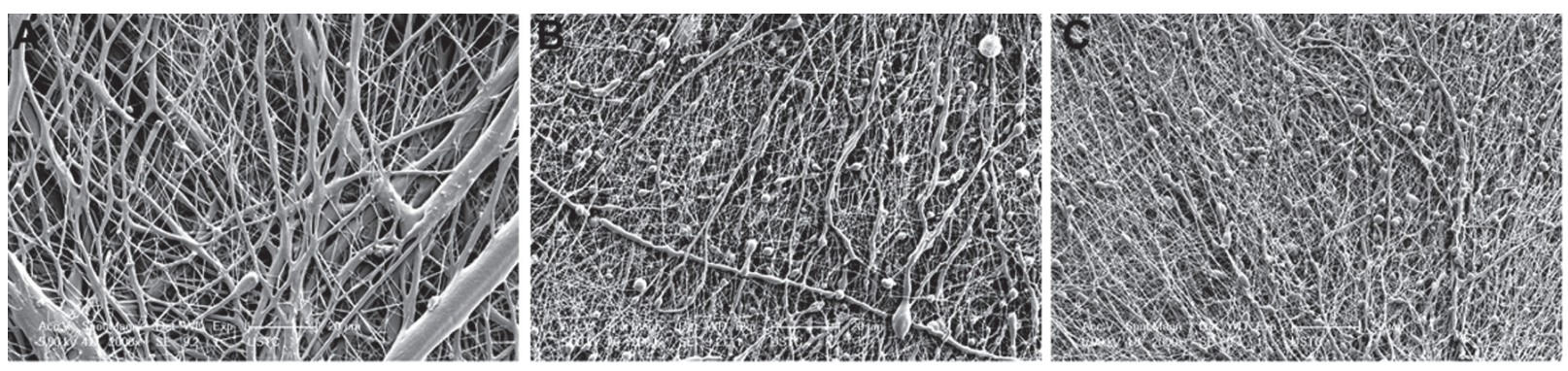

Fig. 1 Scanning electron microscopy images of electrospun nanofibous membrane prepared from different PCL/CS weight ratios.

A) CS/PCL (0:100); B) CS/PCL (50:50); C) CS/PCL (30:70)
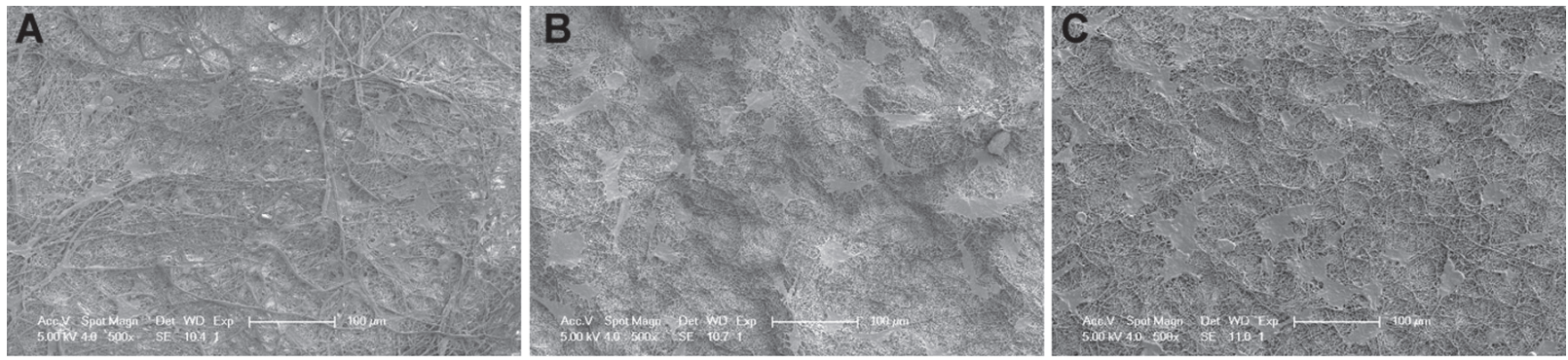

Fig. 2 Scanning electron microscopy images of rBMSCs grown on PCL/CS membrane on day 4 after incubation. A) CS/PCL (0:100); B) CS/PCL (50:50); C) CS/PCL (30:70) (500×)
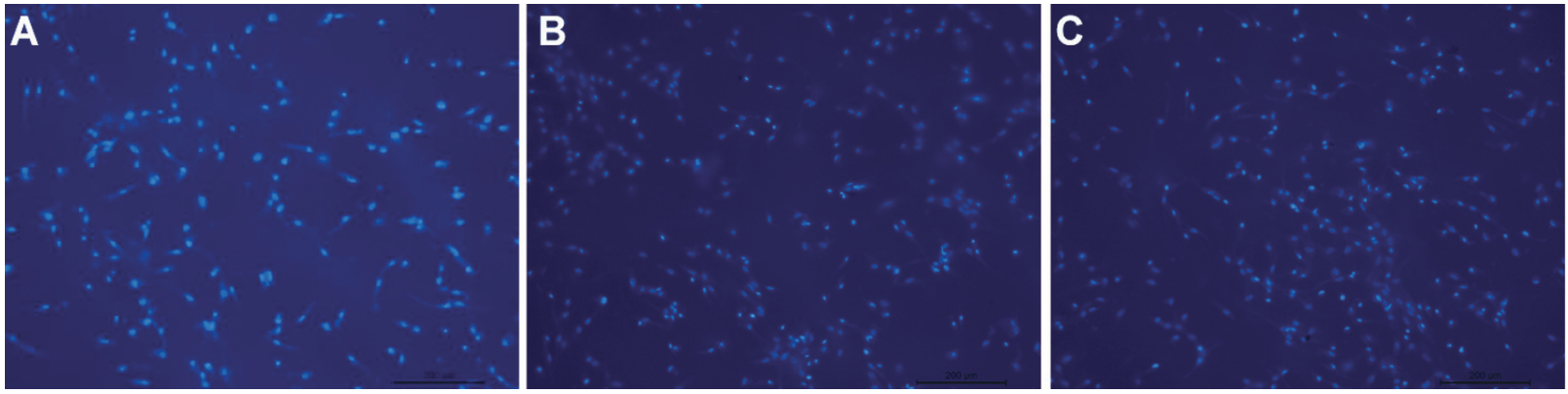

Fig. 3 DAPI fluorescent staining of rBMSCs on PCL/CS membrane on day 7 after incubation. A) CS/PCL (0:100); B) CS/PCL (50:50); C) CS/PCL (30:70)

seeded in the membranes after the fourth day. SEM shows that cells were polygonal, spindle and irregular shape and had more protrusion on the surface of membranes, indicating that the rBMSCs had good adhesion and spreading ability on the surface of three materials. In addition to this, we also observed that both CS/PCL (30:70, 50:50) membrane had more adhered cells than the CS/PCL $(0: 100)$ membrane, indicating that the BMSCs adhered and grew on chitosan/PCL nanofibrous membranes more efficiently than pure PCL.

The proliferation of $r$ BMSCs on the membranes

Figure 3 shows a representation of fluorescent micrograph (DAPI) of rBMSCs on different membranes at day 4 after cell seeding. Generally, cell numbers increased steadily on all membranes during the culture time. Nevertheless, the amount of cells of the membrane CS/PCL (30:70) was evidently higher compared to that on the CS/PCL $(0: 100)$ membrane or on the CS/PCL (50:50) membrane, after 7 days of culture. This result was further confirmed by MTT assay. Figure 4 shows the membrane CS/PCL (30:70) promoted more rBMSCs proliferation than CS/PCL (50:50) group and the CS/ PCL (0:100) membrane.

Osteogenic differentiation of rBMSCs on the membranes To detect whether nanofibrous membrane could influence the osteogenic differentiation of rBMSCs, ALP activity was examined. The results indicated that the ALP activity of rBMSCs in all groups increased 
respectively with the increase of culture time, and the ALP activity on day 14 were remarkably higher than that on day 7 (Table 1). In more details, after culture with membranes for 7 days, the ALP activity of rBMSCs in all three kinds of nanofibrous membranes were

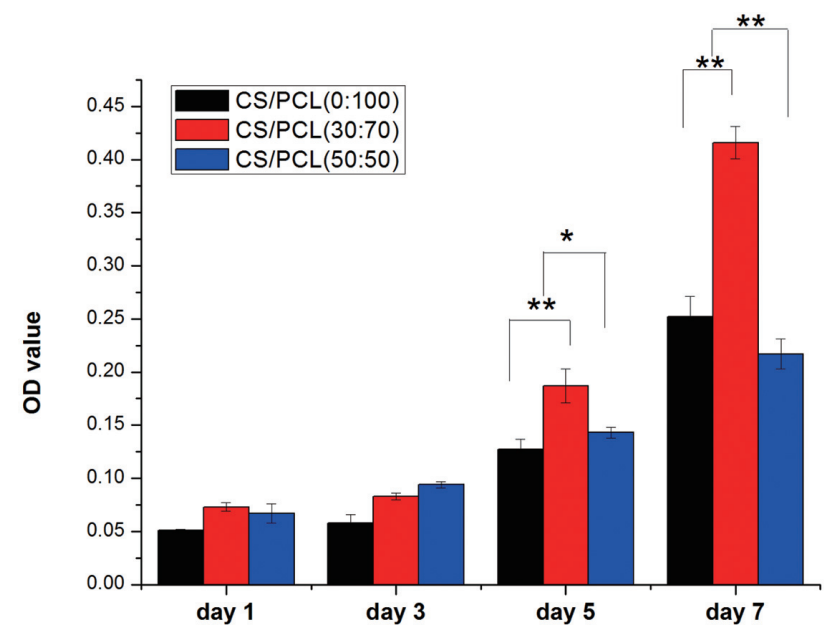

Fig. 4 Analysis of rBMSCs proliferation on the different nanofibrous membranes by MTT assay.

Bar represents mean \pm standard deviation $(n=8)$, ${ }^{*} p<0.05,{ }^{* *} p<0.01$ significant difference between two groups. significantly higher than the untreated rBMSCs, but there were no significant differences in ALP activity among the three materials. Interestingly, on the 14th day, the ALP activity in the CS/PCL (30:70) and CS/ PCL (50:50) group was dramatically higher than of the CS/PCL (0:100) group, indicating a progressive increase of the ALP activity with increase of the CS content. In addition to this, mineral deposition of rBMECs cultured in three nanofibrous membranes was analyzed by AS-R assay and the result showed that the three nanofibrous membrane could induce BMSCs to produce large amounts of red dye calcium nodules after 21 days, and when the CS was added, more calcium mineral deposits were formed (Fig. 5).

After having confirmed that the nano fibrous membranes could induce rBMSCs osteogenic differentiation by using ALP and BCA, we wanted to further verify these results at the protein level. RUNX2 and OCN are osteogenic-related proteins that are involved in osteogenic differentiation. As shown in Fig. 6 , on the 14th day, the RUNX-2 expression was more obvious in the CS/PCL (30:70) than in other two materials and the negative group. Similarly, the expression of OCN showed an increase when the CS was added into the PCL membrane, and reached the most value in the CS/PCL (30:70) group.

Penetration characteristic for fibroblasts of the membranes As shown in Fig. 7, all three electrospun chitosan/PCL

Table 1 ALP activity of rBMECs cultured in three kinds of nanofibrous membranes

\begin{tabular}{ccc}
\hline Group & ALP (the seven day) & ALP (the fourteenth day) \\
\hline A (CS:PCL=0:100) & $3.16 \pm 0.68^{*}$ & $7.45 \pm 0.49^{*}$ \\
B (CS:PCL=50:50) & $4.98 \pm 0.51^{* *}$ & $13.65 \pm 0.65^{* *}$ \\
C (CS:PCL=30:70) & $5.49 \pm 0.61^{* *}$ & $14.16 \pm 0.76^{* *}$ \\
Control group & $2.08 \pm 0.49$ & $4.37 \pm 0.73$ \\
\hline
\end{tabular}

CS: Chitosan, PCL: Polycaprolactone; $n=3,{ }^{*} p<0.05,{ }^{* *} p<0.01$ v.s the rBMECs seeded in 24-well plates without nanofibrous membranes as the negative control.
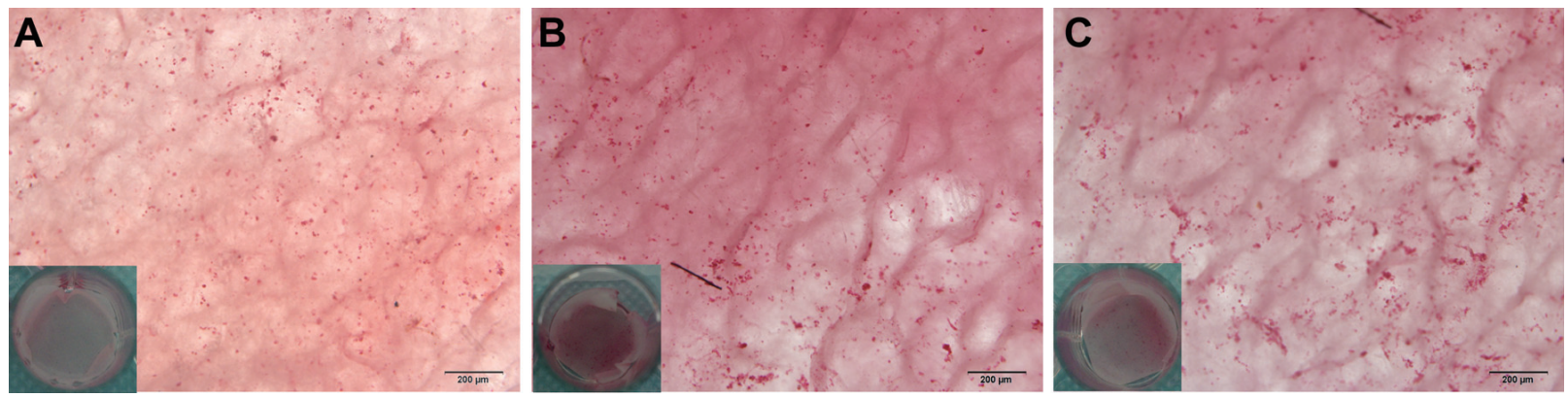

Fig. 5 Alizarin red staining of calcium nodules of rBMSCs grown on PCL/CS membrane on day 21 after incubation.

A) CS/PCL (0:100); B) CS/PCL (50:50) ; C) CS/PCL (30:70) 


\section{Control $\quad 0: 100 \quad 50: 50 \quad 30: 70$}

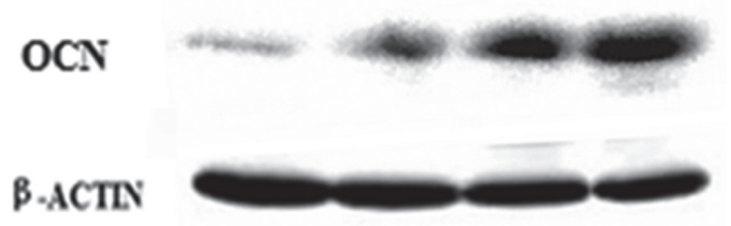

RUNX-2

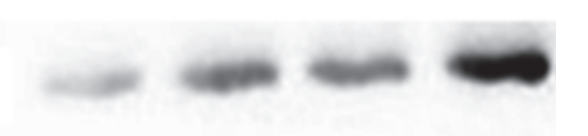

\section{B.ACTIN}

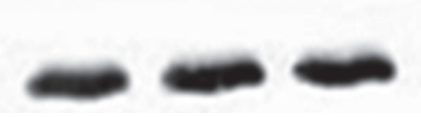

Fig. 6 RUNX-2 and OCN protein expression of rBMSCs grown on PCL/CS membrane on day 14 after incubation.

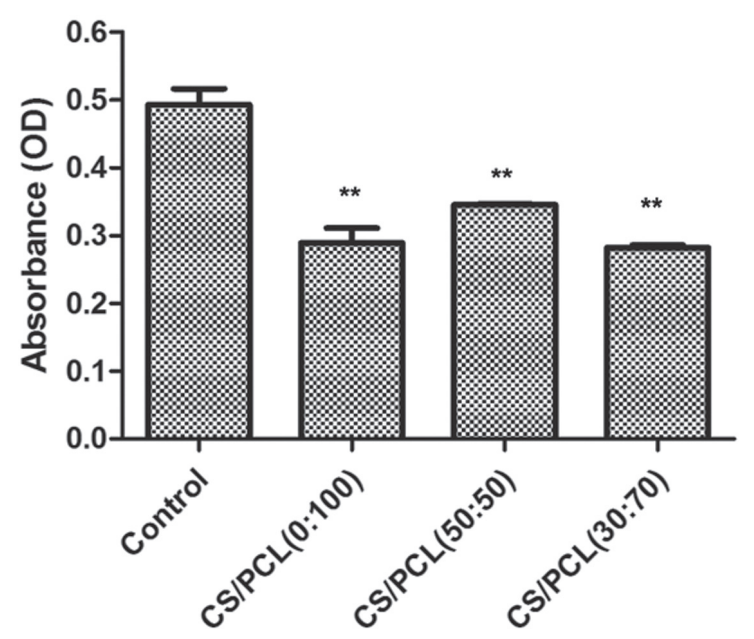

Fig. 7 Penetration of fibroblasts through nanofibrous membranes after $24 \mathrm{~h}$.

The OD values of migrated cells in the lower chamber was determined by CCK- 8 assays. The transwell insert without the nanofibrous membrane was used as the control. ${ }^{* *} p<0.01$ compared with the control. Data are expressed as mean \pm SD $(n=6)$.

nanofibrous membrane could significantly reduce the number of penetrated cells compared to the control (a porous membrane, transwell cell culture inserts, Corning), and the order of penetrated cells through three membranes was as follow: transwell insert membrane $>$ CS/PCL $\quad(50: 50)>C S / P C L \quad(30: 70)>C S / P C L$ (0:100). Indicating three novel membranes could block cell penetration at some extents.

\section{DISCUSSION}

Guided tissue regeneration (GTR) technology has been widely used in repair of various types of bone defects, such as severe periodontal lesions, or immediate dental implant surgery. However, the ideal resorbable GTR membrane has yet to be developed. Chitosan has good biocompatibility, biodegradability, bone regeneration, antimicrobial resistance and is a hydrophilic noncytotoxic natural material ${ }^{18-20)}$. However, the mechanical propertie of chitosan is weak, it has poor plasticity which does not make it an optimum material in the production process of regeneration membrane. PCL has good mechanical properties, easy processing and plasticity, which has widely gathered attention, but studies have also shown that it still has some deficiencies, such as slow degradation rate, high hydrophobicity and poor biocompatibility. Some essays stated clearly that a few natural biomaterials, including gelatin, chitosan, and lecithin could strengthen the hydrophilicity and biocompatibility of $\mathrm{PCL}^{21}$. Moreover, in periodontal tissues engineering, we can make use of periodontal ligament cells (PDL) cells, cementoblasts, BMECs and periosteal cells and so on ${ }^{22}$. Due to the fact that PDL cells were very difficultly sourced and the successful rate of periodontal ligament cells culture in vitro is also very low, and BMSCs have good proliferation and differentiation, osteogenic capability and stable performance characteristics, thus, the rBMECs were chosen in the present study. Therefore, this study mixed together the chitosan and polycaprolactone with different mass ratio, while allowed us to combine their advantages and overcome their disadvantages. Afterwards, the nanofibrous membranes were prepared by electrospinning. Finally, rBMSCs were adhered to the membranes. We observed the adhesion, proliferation and osteogenic properties of BMSCs, and explored whether the nano fibrous membranes meet the requirements for periodontal tissue engineering.

The morphology of electrospun nanofibers is highly influenced by various parameters such as applied voltage, distance between the needle tip and collector and especially properties of polymer solutions including surface tension, viscosity and the nature of solvent ${ }^{23,24)}$. In this study, when the weight ratios of CS/PCL was $0: 100$, we only saw the fibers and no nodules were observed (Fig. 1A). When a portion of CS was added to it, the PCL/CS ratio changed to CS/PCL (50:50), we also examined fibers and found that there was a large amount of nodules in the fibers (Fig. 1B). As the proportion of CS/PCL changes to 30:70, the size of the nodules became smaller and uniform (Fig. 1C). In addition to nodules formation, we also observed that the fibers became thickening in the CS/PCL (50:50) group, suggesting higher porous structures formed with the increase of CS addition. Highly porous structures of membranes are desirable since a high degree of porosity impart large surface area and make more spaces for cell adhesion and migration, on the other hand, higher porous structures of nanofibrous membrane could reduce its cell barrier 
function. Subsequently, the cell adhesion on the three kinds of nanofibrous membrane was measured by SEM analysis. We found that cell numbers of rBMECs increased steadily on all three membranes during the culture time, and evidently higher compared to the control (the rBMECs seeded in cell culture plates). Moreover, the cell numbers of the membrane at a CS/ PCL ratio of 30:70 was higher than that of the membrane with a CS/PCL ratio of $0: 100$ or with a CS/PCL ratio of 50:50 (Fig. 2). In the MTT experiment, three nanofibrous membranes can promote the proliferation of BMSCs after1, 3, 5 and 7 days, differences in OD values at each time point were statistically significant $(p<0.05)$, and showed an increased expression with time trend. At the 1, 3, 5 and 7 days, OD value expression for $\mathrm{CS} /$ PCL (30:70)>CS/PCL (50:50)>CS/PCL (0:100)>control, and the difference was statistically significant $(p<0.05)$. The data showed that nanofibrous membrane of CS/ PCL (30:70) promoted better growth of BMSCs than other groups. It could be attributed to amino groups on the CS/PCL composite membrane which impart more hydrophilic sites than on the CS/PCL (0:100) membrane and the nanofibers of CS/PCL (30:70) with fewer nodules compared to CS/PCL (50:50), which results in more suitable conditions for cell growth and proliferation. Gupta et al. demonstrated that Schwann cells preferred to attach to PCL/gelatin scaffolds with a nanofibrous membrane surface than to PCL scaffolds with a hydrophobic surface ${ }^{14}$. Moreover, an ideal barrier membrane should prevent penetration of epithelial cells or fibroblasts into the bone defect and thus allow for gradual growth and reconstruction of the bone tissue. Therefore, penetration characteristic for fibroblasts of the membranes was measured in this study. By creating a serum concentration gradient across the cell insert during in vitro cell culture, the number of viable cells migrated to the lower chamber through the cell insert was determined by the CCK- 8 assay. Our data demonstrated that with the increase of chitosan content, the cell barrier function of CS/PCL membrane was weakened, which may be due to the increased porous structure of CS/PCL (50:50) nanofibrous membrane (Fig. 7).

Since ALP is considered to be an important marker of the differentiation of osteoblasts at a relatively early bone forming stage, it has been widely used to evaluate the performance of biomaterials in the bone formation process. Studies have shown that osteoblasts not only secrete collagen and other glycoproteins in bone matrix, but are also involved in the metabolism of calcium. Consequently, it is pivotal in the bone defect repair process. In the experiment of ALP and Alizarin red detection, we discovered that the nanofibrous membranes could promote osteogenesis of rBMSCs. More specifically, three kinds of nanofibrous membranes at 7 and 14 days could cause the alkaline phosphatase activity (ALP) of BMSCs to increase and the expression was gradually increased over time during the process of induction. Moreover, among the three groups, ALP activity was as follows: CS/PCL (30:70)>CS/PCL (50:50)>CS/PCL (0:100)>control (Table
1). Similarly, both CS/PCL nanofibrous membranes caused a significant increase of extracellular calcium deposition in BMSCs after 21 days (Fig. 5), suggesting CS/PCL nanofibrous membranes had better osteogenic ability for the mineralization induction of BMECs than the pure PCL membrane. On the basis of analyzing ALP activity and mineral deposition, this study further used the proteins levels to evaluate the osteogenic differentiation ability of fiber membrane. Among them, RUNX-2 as a bone cell-specific transcription factor, plays a key role in the osteogenic differentiation pathway ${ }^{25}$. Osteocalcin (OCN) occurs mainly during the mineralization stage and is a sign of mature bone cells. OCN is a sign of bone cells maturity and is involved in bone formation. Western blot was used for the detection of the expression of RUNX-2 and OCN protein. According to the protein bands, we found that the expression of RUNX-2 and OCN of BMECs in the CS/PCL (30:70) group was significantly higher than that in the other two groups. When compared to the ALP tests, the experimental results were the same. This can be explained by the fact that adding chitosan can boost osteogenic differentiation of cells. Recent studies have shown that when mice osteoblasts are placed in the chitosan nanofiber scaffold, the stent material promoted osteogenic differentiation of the cells via upregulation of the Runx2/osteocalcin/alkaline phosphatase signaling pathway ${ }^{26)}$. Chitosan has been widely used as a carrier in the field of tissue engineering. The material itself is made by the electrostatic spinning technique for preparing three-dimensional fiber diameter of nano fibers which are similar to cell size and mimics the natural environment of the extracellular matrix, promotes adhesion and cell differentiation ${ }^{27)}$. In our experiment, the results of CS/PCL (30:70) is better than that of CS/PCL (50:50), although groups of CS/ PCL (50:50) with chitosan content is conducive to the proliferation and differentiation of BMSCs, however, the structures of nano fibrous membranes when chitosan was added were not optimum and were not conducive to cell adhesion and proliferation. Therefore, the appropriate proportion of chitosan and polycaprolactone is particularly important to the enhanced property of these new nano fibrous membranes.

\section{CONCLUSION}

Our data demonstrated that the CS/PCL (30:70) nanofibrous membrane promoted an increased rBMSCs proliferation when compared to the CS/PCL (50:50) membrane and pure PCL (0:100) membrane. The highest ALP activity and extracellular calcium deposit were observed on the CS/PCL (30:70) nanofibrous membrane, followed by the CS/PCL (50:50) and pure PCL nanofibrous membrane. The expression of osteocalcin (OCN) and Runx2 were also significantly higher on the CS/PCL (30:70, 50:50) nanofibrous membrane as compared to the pure PCL nanofibrous membrane. Furthermore, the pure PCL and CS/PCL (30:70) nanofibrous membrane had stronger barrier 
function than the CS/PCL (50:50). In conclusion, the electrospun CS/PCL nanofibrous membrane developed in this study was found to be a biocompatible material that could stimulate rBMSCs proliferation and osteogenic differentiation, and the best result was observed in the chitosan/PCL (30:70) group, suggesting that the novel nanofibrous composite membrane of CS/ PCL has an interesting potential and prospect as use for guided periodontal regeneration material.

\section{ACKNOWLEDGMENTS}

This study was supported by Grants for Scientific Research of BSKY (No. XJ201108) from Anhui Medical University and National Natural Science Foundation of China (No. 81201191).

\section{REFERENCES}

1) Schröder NW, Meister D, Wolff V, Christan C, Kaner D, Haban V, Purucker P, Hermann C, Moter A, Göbel UB, Schumann RR. Chronic periodontal disease is associated with single-nucleotide polymorphisms of the human TLR-4 gene. Genes Immun 2005; 6: 448-451.

2) Simion M, Baldoni M, Rossi P, Zaffe D. A comparative study of the effectiveness of e-PTFE membranes with and without early exposure during the healing period. Int J Periodontics Restorative Dent 1994; 14: 166-180.

3) Liao S, Wang W, Uo M, Ohkawa S, Akasaka T, Tamura K, Cui F, Watari F. A three-layered nano-carbonated hydroxyapatite/ collagen/PLGA composite membrane for guided tissue regeneration. Biomaterials 2005; 26: 7564-7571.

4) Song JH, Kim HE, Kim HW. Collagen-apatite nanocomposite membranes for guided bone regeneration. J Biomed Mater Res B Appl Biomater 2007; 83: 248-257.

5) Lowe B, Venkatesan J, Anil S, Shim MS, Kim SK. Preparation and characterization of chitosan-natural nano hydroxyapatitefucoidan nanocomposites for bone tissue engineering. Int $\mathrm{J}$ Biol Macromol 2016; 93: 1479-1487.

6) Muzzarelli RA, Mattioli-Belmonte M, Tietz C, Biagini R, Ferioli G, Brunelli MA, Fini M, Giardino R, Ilari P, Biagini G. Stimulatory effect on bone formation exerted by a modified chitosan. Biomaterials 1994; 15: 1075-1081.

7) Prabhakaran MP, Venugopal JR, Chyan TT, Hai LB, Chan CK, Lim AY, Ramakrishna S. Electrospun biocomposite nanofibrous scaffolds for neural tissue engineering. Tissue Eng Part A 2008; 14: 1787-1797.

8) Shin SY, Park HN, Kim KH, Lee MH, Choi YS, Park YJ, Lee YM, Ku Y, Rhyu IC, Han SB, Lee SJ, Chung CP. Biological evaluation of chitosan nanofiber membrane for guided bone regeneration. J Periodontol 2005; 76: 1778-1784.

9) Chang KY, Cheng LW, Ho GH, Huang YP, Lee YD. Fabrication and characterization of poly(gamma-glutamic acid)-graftchondroitin sulfate/polycaprolactone porous scaffolds for cartilage tissue engineering. Acta Biomater 2009; 5: 19371947.

10) Venugopal J, Zhang YZ, Ramakrishna S. Fabrication of modified and functionalized polycaprolactone nanofibre scaffolds for vascular tissue engineering. Nanotechnology 2005; 16: 2138-2142.

11) Fukunishi T, Best CA, Sugiura T, Shoji T, Yi T, Udelsman B, Ohst D, Ong CS, Zhang H, Shinoka T, Breuer CK, Johnson J, Hibino N. Tissue-engineered small diameter arterial vascular grafts from cell-free nanofiber PCL/Chitosan scaffolds in a sheep model. PLoS One 2016; 11: e0158555.

12) Bai MY, Chou TC, Tsai JC, Yu WC. The effect of active ingredient-containing chitosan/polycaprolactone nonwoven mat on wound healing: in vitro and in vivo studies. J Biomed Mater Res A 2014; 102: 2324-2333.

13) Shao HJ, Lee YT, Chen CS, Wang JH, Young TH. Modulation of gene expression and collagen production of anterior cruciate ligament cells through cell shape changes on polycaprolactone/ chitosan blends. Biomaterials 2010; 31: 4695-4705.

14) Chen H, Fan X, Xia J, Chen P, Zhou X, Huang J, Yu J, Gu P. Electrospun chitosan-graft-poly (varepsilon-caprolactone)/ poly (varepsilon-caprolactone) nanofibrous scaffolds for retinal tissue engineering. Int $J$ Nanomedicine 2011; 6: 453461.

15) Neves SC, Moreira Teixeira LS, Moroni L, Reis RL, Van Blitterswijk CA, Alves NM, Karperien M, Mano JF. Chitosan/ poly(epsilon-caprolactone) blend scaffolds for cartilage repair. Biomaterials 2010; 32: 1068-1079.

16) Jing X, Mi HY, Wang XC, Peng XF, Turng LS. Shishkebab-structured poly(epsilon-caprolactone) nanofibers hierarchically decorated with chitosan-poly(epsiloncaprolactone) copolymers for bone tissue engineering. ACS Appl Mater Interfaces 2015; 7: 6955-6965.

17) Chen CH, Chen SH, Shalumon KT, Chen JP. Prevention of peritendinous adhesions with electrospun polyethylene glycol/polycaprolactone nanofibrous membranes. Colloids Surf B Biointerfaces 2015; 133: 221-230.

18) Ikinci G, Senel S, Akincibay H, Kas S, Ercis S, Wilson CG, Hincal AA. Effect of chitosan on a periodontal pathogen Porphyromonas gingivalis. Int J Pharm 2002; 235: 121-127.

19) Kim KW, Thomas RL, Lee C, Park HJ. Antimicrobial activity of native chitosan, degraded chitosan, and O-carboxymethylated chitosan. J Food Prot 2003; 66: 1495-1498.

20) Klokkevold PR, Fukayama H, Sung EC, Bertolami CN. The effect of chitosan (poly-N-acetyl glucosamine) on lingual hemostasis in heparinized rabbits. J Oral Maxillofac Surg 1999; 57: 49-52.

21) Dhandayuthapani B, Krishnan UM, Sethuraman S. Fabrication and characterization of chitosan-gelatin blend nanofibers for skin tissue engineering. J Biomed Mater Res B Appl Biomater 2010; 94: 264-272.

22) Yamada Y, Ueda M, Hibi H, Baba S. A novel approach to periodontal tissue regeneration with mesenchymal stem cells and platelet-rich plasma using tissue engineering technology: A clinical case report. Int $\mathrm{J}$ Periodontics Restorative Dent 2006; 26: 363-369.

23) Shuakat MN, Lin T. Recent developments in electrospinning of nanofiber yarns. J Nanosci Nanotechnol 2014; 14: 13891408.

24) Jiang H, Fang D, Hsiao BS, Chu B, Chen W. Optimization and characterization of dextran membranes prepared by electrospinning. Biomacromolecules 2004; 5: 326-333.

25) Pratap J, Galindo M, Zaidi SK, Vradii D, Bhat BM, Robinson JA, Choi JY, Komori T, Stein JL, Lian JB, Stein GS, van Wijnen AJ. Cell growth regulatory role of Runx2 during proliferative expansion of preosteoblasts. Cancer Res 2003; 63: 5357-5362.

26) Ho MH, Yao CJ, Liao MH, Lin PI, Liu SH, Chen RM. Chitosan nanofiber scaffold improves bone healing via stimulating trabecular bone production due to upregulation of the Runx2/ osteocalcin/alkaline phosphatase signaling pathway. Int J Nanomedicine 2015; 10: 5941-5954.

27) Lim SH, Mao HQ. Electrospun scaffolds for stem cell engineering. Adv Drug Deliv Rev 2009; 61: 1084-1096. 